Pacific Journal of Mathematic 


\section{MULTIPLIER ALGEBRAS OF BIORTHOGONAL SYSTEMS}

\section{Raymond J. McGivney and William Ruckle}

Let $\left\{e_{i}, E_{i}\right\}$ be a total biorthogonal system in a linear topological space $X$. The multiplier algebra of $X$ with respect to $\left\{e_{i}, E_{i}\right\}$ written $M(X)$ is the set of all scalar sequences $\left(t^{(i)}\right)$ such that for each $x \in X$ there is $y \in X$ with

$$
E_{i}(y)=t^{(i)} E_{i}(x) \text {. }
$$

The form of $M(X)$ is determined when $\left\{e_{i}, E_{i}\right\}$ is a norming complete biorthogonal system in a Banach space or a basis in a complete barreled space. It is shown that a sequence space is the multiplier algebra for a basis in a Banach space if and only if it is a $\gamma$-perfect $B K$-algebra.

A biorthogonal system is a double sequence $\left\{e_{i}, E_{i}\right\}$ with each $e_{i}$ in a locally convex space $X$ and each $E_{i}$ a continuous linear functional on $X$ (i.e., in $X^{*}$ ) which satisfies the relationship

$$
E_{i}\left(e_{j}\right)=\delta_{i j}(\text { Kronecker } \delta) i, j=1,2, \cdots \text {. }
$$

The biorthogonal system is total if $\left\{E_{i}\right\}$ is total on $X$; that is, $E_{i}(x)=0$ for each $i$ implies $x=0$. If $\left\{e_{i}, E_{i}\right\}$ is a total biorthogonal system then the space $X$ can be identified with the space of all sequences $\left(E_{i}(x)\right)$ by means of the natural correspondence $x$ to $\left(E_{i}(x)\right)$. Under this correspondence $e_{i}$ becomes the $i$ th coordinate vector, the sequence which has a one in the $i$ th coordinate and 0 's elsewhere and $E_{i}$ becomes the $i$ th coordinate functional, the functional whose value on the sequence $\left(x^{(1)}, x^{(2)}, \ldots\right)$ is $x^{(i)}$. This identification will be assumed whenever a total biorthogonal system is considered.

DeFinition 1.1. Let $\left\{e_{i}, E_{i}\right\}$ be a total biorthogonal system in a locally convex space $X$. A scalar sequence

$$
t=\left(t^{(1)}, t^{(2)}, \cdots\right)
$$

is a multiplier of $X$ with respect to $\left\{e_{i}, E_{i}\right\}$ if for each $x \in X$ there is $y \in X$ for which

$$
E_{i}(y)=t^{(i)} E_{i}(x) \quad i=1,2, \cdots .
$$

The set of all such $t$ is written $M\left(X ; e_{i}, E_{i}\right)$ or simply $M(X)$ and called the multiplier algebra of $X$ (with respect to $\left\{e_{i}, E_{i}\right\}$ ).

In other words $M(X)$ is the set of all $t$ such that $t x \in X$ whenever $x \in X$ 
where $X$ is now considered a sequence space and multiplication of sequences is defined coordinatewise. It is now obvious that $M(X)$ forms a linear algebra of operators from $X$ into $X$; namely the operators which are diagonal with respect to $\left\{e_{i}, E_{i}\right\}$. Multiplication in this algebra is defined coordinatewise. The purpose of this paper is to study the properties of the space $M(X)$ and the possible forms which it can assume with varying hypotheses on $X$ or on $\left\{e_{i}, E_{i}\right\}$. Results of this type were obtained by Yamazaki in [11] and [12] for $\left\{e_{i}\right\}$ a basis in a Banach space. The concept of imultiplier space is implicitly treated in [4].

Throughout this paper it is immaterial whether the scalar field considered consists of the real or complex numbers.

2. Sequence spaces: notation and basic facts. A set of scalar sequences which is closed under coordinatewise addition and scalar multiplication is a sequence space; if it is closed under coordinatewise multiplication as well it will be called a sequence algebra. The $i$ th coordinate vector is written $e_{i}$; the $i$ th coordinate functional, $E_{i}$. If each $E_{i}$ is continuous on a locally convex sequence space (algebra) $X$ and $e_{i} \in X$ for each $i$ then $X$ is called a $K$-space (algebra). If in addition $X$ is an $F$-space (complete metric linear space) $X$ will be called an $F K$-space or $F K$-algebra as the case may be. If $X$ is a Banach space (algebra), $X$ will be called a $B K$-space (algebra). Note that in an $F K$-algebra $X$ the functions $x \rightarrow t x$ and $x \rightarrow x t$ are continuous in $x$ for fixed $t$ by the continuity of the coordinate functionals and the closed graph theorem. This is enough to conclude that a $B K$-algebra is a Banach algebra without identity. See p. 860 and 861 of [3].

The following are well known sequence spaces. For additional discussion see Chapter IV of [2], [5] or p. 289 of [10]:

$\omega$ sometimes called $s$ is the set of all scalar sequences. Endowed with the topology of coordinatewise convergence it is an $F K$-algebra.

$\varphi$ is the linear span of $\left\{e_{i}\right\}$ in $\omega$, i.e., the space of all finitely nonzero sequences.

$l^{1}$ is the set of all sequences $t$ such that

$$
\|t\|=\sum_{i=1}^{\infty}\left|t^{(i)}\right|<\infty
$$

which is a $B K$-space with this norm.

$m$ is the set of all sequences $t$ such that

$$
\|t\|=\sup _{i}\left|t^{(i)}\right|<\infty
$$

which is a $B K$ algebra with this norm. 
$b s$ is the set of all sequences $t$ such that

$$
\|t\|=\sup _{n}\left|\sum_{i=1}^{n} t^{(i)}\right|<\infty
$$

which is a $B K$ space with this norm.

$c s$ is the closed linear span of $\left\{e_{i}\right\}$ in $b s$; it consists of all sequences $t$ such that $\sum_{i=1}^{\infty} t^{(i)}$ converges.

$b v$ is the set of all sequences $t$ such that

$$
\|t\|=\lim _{n}\left|t_{n}\right|+\sum_{i=1}^{\infty}\left|t_{i}-t_{i+1}\right|<\infty
$$

which is a $B K$-algebra with this norm. See $\S 3$ of [4] or p. 3 of [11]. Yamazaki denoted $b v$ by $w$.

Notation 2.1. (a) Let $t, u \in \omega$ be such that $u t \in c s$; the sum $\sum_{i=1}^{\infty} u^{(i)} t^{(i)}$ is denoted by $(u, t)$.

(b) For $A \subseteq \omega$

$A^{\alpha}$, the $\alpha$-dual of $A$ is $\left\{t: u t \in l^{1}, u \in A\right\}$

$A^{\beta}$, the $\beta$-dual of $A$ is $\{t: u t \in c s, u \in A\}$

$A^{\gamma}$, the $\gamma$-dual of $A$ is $\{t: u t \in b s, u \in A\}$.

(c) For $A$ and $B \subseteq \omega$

$A B=\{u v: u \in A, v \in B\}$.

(d) For $t \in \omega$ and $A \subseteq \omega$

$t^{-1} A=\{u \in \omega: t u \in A\}$.

(e) For $A \subseteq \omega$

$A^{\varsigma}=\{t \in \varphi:|(t, u)| \leqq 1, u \in A\}$.

(f) For $A \subseteq \varphi$

$A^{\omega}=\{t \in \omega:|(t, u)| \leqq 1, u \in A\}$.

(g) For $X$ aK-space, $X^{\circ}$ is the space of all sequences $\left(f\left(e_{i}\right)\right)$ as $f$ ranges over $X^{*}$. Note that for $t \in X^{\delta}$ with $t^{(i)}=f\left(e_{i}\right), E_{i}(t)=t^{(i)}=f\left(e_{i}\right)$.

Gamma-perfect $B K$-spaces can be constructed by means of sequential norms. A sequential norm (s.n.) is a function $P$ from $\omega$ into $R^{*}$ which is an extended norm and in addition satisfies the condition

$$
P(x)=\sup _{n} P\left(\sum_{i=1}^{n} x^{(i)} e_{i}\right) x \in \omega .
$$

If

$$
0<\inf _{n} P\left(e_{n}\right) \leqq \sup _{n} P\left(e_{n}\right)<\infty
$$

$P$ is a proper sequential norm (p.s.n.). For $P$ a s.n, $S_{P}$ is the set of all $x \in \omega$ for which $P(x)<\infty$ endowed with the topology determined by $P \varepsilon$. The closed linear span of $\left\{e_{1}, e_{2}, \cdots\right\}$ in $S_{P}$ is denoted by $S_{P}^{0}$. The 
following proposition contains information about sequential norms which was derived in [7] and [8] and which we shall use in $\$ 6$.

Proposition 2.2. (a) If $P$ is a s.n. $S_{P}$ is a $\gamma$-perfect $B K$-space. If $X$ is a $\gamma$-perfect $B K$-space there is an s.n. $P$ such that $X=S_{P}$.

(b) If $P$ is a s.n. the function $P^{\prime}$ given by

$$
P^{\prime}(x)=\sup \left\{\sup _{n}\left|\sum_{i=1}^{n} x^{(i)} y^{(i)}\right|: P(y) \leqq 1\right\}
$$

is a s.n. and $P^{\prime \prime}=P$. If $P$ is a p.s.n. so is $P^{\prime}$.

(c) $\left(S_{P}^{0}\right)^{\delta}=S_{P \prime}$ and $\left(S_{P \prime}^{0}\right)^{\delta}=S_{P}$.

(d) An s.n. $P$ is a p.s.n. if and only if $l^{1} \cong S_{P} \subseteq m$.

3. Preliminary results.

Proposition 3.1. If $\left\{e_{i}, E_{i}\right\}$ is a total biorthogonal system in $X$ $(3-1)$

$$
M(X)=\cap\left\{y^{-1} X: y \in S\right\}
$$

where $S$ is any absorbing subset of $X$.

Proof. Let $R$ denote the set on the right of (3-1). If $t \in R$ and $x \in X$ there is $a>0$ such that $a x \in S$. Thus at $x \in X$ so that $t x \in X$ which implies $t \in M(X)$. If $t \in M(X)$ then $t x \in X$ for every $x \in X$ so in particular for every $x \in S$.

A complete biorthogonal system is a total biorthogonal system $\left\{e_{i}, E_{i}\right\}$ on $X$ such that $\mathrm{sp}\left\{e_{i}\right\}(=\varphi)$, the linear span of $\left\{e_{i}\right\}$ is dense in $X$.

Proposition 3.2. Let $\left\{e_{i}, E_{i}\right\}$ be a complete biorthogonal system in $X$.

(a) For each $t \in M(X)$, the mapping $x \rightarrow t x$ is a closed linear operator from $X$ into $X$.

(b) The set $M_{c}(X)$ of all $t \in M(X)$ for which $x \rightarrow t x$ is continuous is a closed sub-algebra of $\mathscr{L}(X)$ where $\mathscr{L}(X)$ has any topology containing the topology of simple convergence. Here $\mathscr{L}(X)$ denotes the space of continuous operators from $X$ into $X$.

Proof. (a) Obvious.
(b) Define $E_{i} \otimes e_{j}$ on $\mathscr{L}(X)$ by

$$
E_{i} \otimes e_{j}(T)=E_{i}\left(T e_{j}\right) \text {. }
$$

Then $E_{i} \otimes e_{j}$ is a continuous linear functional on $\mathscr{L}(X)$ given the topology of simple convergence. Therefore

$$
S=\cap\left\{\left[E_{i} \otimes e_{j}\right]^{-1}(0): i \neq j\right\}
$$


is closed in this topology and every topology containing it.

The following statement generalizes Theorem 1 of [11].

CoRollaRY 3.3. If $\left\{e_{i}, E_{i}\right\}$ is a complete biorthogonal system in a Banach space $X$ then there is a topology on $M(X)$ which makes it a $B K$ algebra.

Proof. In this case $M_{c}(X)=M(X)$ since the mapping $x \rightarrow t x$ is closed. Thus by $3.2 M(X)$ is a Banach algebra with the norm

$$
\|t\|=\sup \{\|t x\|:\|x\| \leqq 1\} \text {. }
$$

Each $E_{i}$ is continuous on $M(X)$ since

$$
E_{i}(T)=E_{i} \otimes e_{i}\left(T_{t}\right)
$$

where $T_{t}(x)=t x$; and $E_{i} \otimes e_{i}$ is continuous on $\mathscr{L}(X)$.

Proposition 3.4. If $\left\{e_{i}, E_{i}\right\}$ is a total biorthogonal system in a linear topological space $X$ then

$$
M_{c}\left(X ; e_{i}, E_{i}\right) \subseteq M_{c}\left(X_{0} ; e_{i}, E_{i}\right)
$$

where $X_{0}$ is the closed linear span of $\left\{e_{i}\right\}$ in $X$.

Proof. If $t \in M_{c}(X)$ then $t x \in \varphi$ for $x \in \varphi$. Since $\varphi$ is dense in $X_{0}$ and $t$ is continuous $t x \in X_{0}$ for $x \in X_{0}$ so that $t \in M_{c}\left(X_{0}\right)$.

If $\left\{e_{i}, E_{i}\right\}$ is a complete biorthogonal system on $X, X^{*}$ is isomorphic to $X^{\delta}$ under the correspondence of $f$ in $X^{*}$ to $\left(f\left(e_{i}\right)\right)$ in $X^{\delta}$ and $\left\{e_{i}, E_{i}\right\}$ is a total biorthogonal system on $X^{j}$.

Proposition 3.5. If $\left\{e_{i}, E_{i}\right\}$ is a complete biorthogonal system in a locally convex space $X$ then

$$
M_{c}\left(X ; e_{i}, E_{i}\right) \subseteq M\left(X^{i} ; e_{i}, E_{i}\right) .
$$

Proof. If $t \in M_{c}$ for $f \in X^{*}$ let $f_{t}(x)=f(t x), x \in X$. Then $f_{t} \in X^{*}$ and $f_{t}\left(e_{i}\right)=t^{(i)} f\left(e_{i}\right)$ for each $i$ so that $t y \in X^{\delta}$ for $y \in X^{\delta}$.

4. Multiplier algebras of a norming biorthogonal system in a Banach space. A biorthogonal system $\left\{e_{i}, E_{i}\right\}$ in a normed space $X$ is called norming if the topology of $X$ is determined by a norm of the type

$$
\|x\|=\sup \{|f(x)|: f \in A\}
$$


where $A$ is a subset of the linear span of $\left\{E_{i}\right\}$ in $X^{*}$. An equivalent condition is that the above norm be given by

$$
\|x\|=\sup \{|(x, t)|: t \in A\}
$$

where $A$ is a subset of $\varphi$.

If $\left\{e_{i}, E_{i}\right\}$ is a complete biorthogonal system which is norming on $X$ and the norm of $X$ is given by (4-1) it may be assumed that $A$ consists of all sequences $t$ in $\varphi$ for which

$$
|(t, x)| \leqq\|x\| \quad x \in X
$$

Denote by $\hat{X}$ the space of all $x \in \omega$ for which

$$
\|x\|=\sup \{|(x, t)|: t \in A\}<\infty .
$$

The function defined in (4-3) is a norm since $a_{n}^{-1} e^{n} \in A$ for $n=1,2, \cdots$ where $a_{n}$ is the norm of $E_{n}$ as a member of $X^{*}$. With this norm $\hat{X}$ is a $B K$-space in which $X$ is the closed linear span of $\left\{e_{i}\right\}$.

Proposition 4.1. The space $X^{\delta}$ consists of all $y \in \omega$ for which

$$
\|y\|^{\prime}=\sup \left\{|(y, x)|: x \in A^{\varphi}\right\}<\infty .
$$

The correspondence

$$
f \text { to }\left(f\left(e_{i}\right)\right) \quad f \in X^{*}
$$

is an isometry from $X^{*}$ onto $\left(X^{\delta},\|\|^{\prime}\right)$. The correspondence

$$
g \text { to }\left(g\left(e_{i}\right)\right) \quad g \in\left(X_{0}^{\delta}\right)^{*}
$$

is an isometry from $\left(X_{0}^{o}\right)^{*}$ onto $\hat{X}$, where $X_{0}^{\delta}$ denotes the closed linear span of $\left\{e_{i}\right\}$ in $X^{\delta}$.

Proof. The correspondence in (4-4) is clearly well defined and linear.

If $f \in X^{*}$ and $x \in A^{\varphi}$ then $x \in X$ and $\|x\| \leqq 1$. Thus

$$
\begin{aligned}
\left|\left(f\left(e_{i}\right), x\right)\right| & =\left|\sum_{i} x^{(i)} f\left(e_{i}\right)\right| \\
& =|f(x)| \leqq\|f\|
\end{aligned}
$$

so that

$$
\left\|\left(f\left(e_{i}\right)\right)\right\|^{\prime} \leqq\|f\| \text {. }
$$

If $y \in X^{\delta}$ define $f$ on $\{\varnothing,\|\|\}$ by

$$
f(x)=(y, x) .
$$


Then $f$ is a bounded linear functional on $\{\varphi,\|\|\}$ for which

$$
|f(x)| \leqq\|y\|^{\prime} x \in \varphi,\|x\| \leqq 1
$$

because

$$
A^{\circ}=\{x \in \varphi:\|x\| \leqq 1\} .
$$

Since $\varphi$ is dense in $X, f$ can be continuously extended to $X$ with

$$
\|f\| \leqq\|y\|^{\prime} \text {. }
$$

For this extended $f$

$$
\left(f\left(e_{i}\right)\right)=\left(y, e_{i}\right)=y_{i} \quad i=1,2, \cdots .
$$

Therefore, the correspondence in (4-4) is an isometry.

That (4-5) is an isometry from $\left(X_{0}^{\delta}\right)^{*}$ onto $\hat{X}$ will follow from an analogous argument if it is shown that

$$
A^{\varphi \varphi}=A \text {. }
$$

When $A$ has the form given by (4-2).

That $A^{\varphi \varphi} \supseteqq A$ is clear, if $z \in A^{\varphi \varphi}$ then

$$
|(z, x)| \leqq 1 \quad x \in A^{c}
$$

but $A^{\varphi}$ is dense in the unit ball of $X$ so that

$$
|(z, x)| \leqq 1 \quad x \in X,\|x\| \leqq 1 .
$$

Thus if

$$
f(x)=(z, x) \quad x \in X
$$

we have $\|f\| \leqq 1$ and

$$
f\left(e_{i}\right)=z_{i} \quad i=1,2, \cdots
$$

so that $z \in A$. It is here that the assumption that $\left\{e_{i}, E_{i}\right\}$ is norming was used.

THEOREM 4.2. If $\left\{e_{i}, E_{i}\right\}$ is a norming complete biorthogonal system in the Banach space $X$ then $M(X)$ is of the form

$$
\mathbf{U}_{n=1}^{\infty} n\left(A A^{\omega}\right)^{\omega}
$$

where $A$ is a coordinatewise bounded subset of $\varphi$ which contains a multiple of $e_{i}$ for each $i$.

Proof. Let $A$ be given by (4-2) and let $Z$ denote the sequence space (4-6). 
By 3.4, 3.5 and the fact that $M_{c}(Y)=M(Y)$ for $Y$ a Banach space we have

$$
M(X) \subseteq M\left(X^{o}\right) \subseteq M\left(X_{0}^{j}\right) \subseteq M(\hat{X}) \subseteq M(X)
$$

so that $M(X)$ and $M(\hat{X})$ are equal. It will be shown that $M(\hat{X})=Z$. Suppose $t \in M(\hat{X})$, then there is $k$ such that

$$
\|t x\| \leqq k\|x\| \quad x \in \hat{X} \text {. }
$$

If $s \in A$ and $x \in A^{\omega}$

$$
|(s x, t)|=|(s, t x)| \leqq k
$$

so that

$$
t \in k\left(A A^{\omega}\right)^{\omega} \cong Z
$$

If $z \in Z$ and $x \in \hat{X}, x /\|x\| \in A^{\omega}$ so if $n$ is such that $z \in n\left(A A^{\omega}\right)^{\omega}$

$$
|(t, z x)|=|(t x, z)| \leqq n\|x\|
$$

for $t \in A$. Therefore $z x \in \hat{X}$.

Question. If $\left\{e_{i}, E_{i}\right\}$ is a complete biorthogonal system in the Banach space $X$ and $M(X)$ has the form (4-6) is $\left\{e_{i}, E_{i}\right\}$ norming?

5. Multiplier algebras of bases. A biorthogonal system $\left\{e_{i}, E_{i}\right\}$ in a linear topological space $X$ is a (Schauder) basis for $X$ if

$$
x=\sum_{i=1}^{\infty} E_{i}(x) e_{i} \quad x \in X .
$$

It is an unconditional basis if the convergence in (5-1) is unconditional. If $X$ is a l.c.s. $\left\{e_{i}, E_{i}\right\}$ is an absolute basis if the convergence in (5-1) is absolute, i.e., if

$$
\sum_{i=1}^{\infty}\left|E_{i}(x)\right| p\left(e_{i}\right)<\infty
$$

for each $x \in X$ and each continuous seminorm $p$ on $X$. It is clear that an absolute basis is unconditional.

The proofs of 5.1, 5.2, and 5.3 are omitted since these statements are essentially known. See p. 205 of [10] and Propositions 4 and 5 of [1].

Lemma 5.1. For $\left\{e_{i}, E_{i}\right\}$ a complete biorthogonal system in a barreled 1.c.s. $X$ it is always true that $X^{r} \cong X^{\delta}$.

Lemma 5.2. For $\left\{e_{i}, E_{i}\right\}$ a basis in a locally convex space $X, X^{\delta} \subseteq X^{\beta}$. 
Proposition 5.3. For $\left\{e_{i}, E_{i}\right\}$ a complete biorthogonal system in a barreled locally convex space $X$ the following are equivalent.

(a) $\left\{e_{i}, E_{i}\right\}$ is a basis for $X$.

(b) $X^{\delta}=X^{\beta}$.

(c) $X^{\delta}=X^{\gamma}$.

THEOREM 5.4. If $\left\{e_{i}, E_{i}\right\}$ is a basis of a complete barreled space $X$ then

$$
M_{c}(X)=M(X)=\left(X X^{\beta}\right)^{\beta}=\left(X X^{\gamma}\right)^{\gamma}
$$

Proof. Suppose $t \in M(X), x \in X$ and $y \in X^{\beta}$. Then $t x \in X$ so that txy $\in$ cs by 5.1 which implies $t \in\left(X X^{\beta}\right)^{\beta}$.

Let $P$ denote the family of all continuous seminorms on $X$. Let $\hat{X}$ be the linear space of all $x \in \omega$ such that

$$
p^{\prime}(x)=\sup _{n} p\left(\sum_{i=1}^{n} x^{(i)} e_{i}\right)<\infty, p \in P .
$$

Since $X$ is barreled, $p^{\prime}$ restricted to $X$ is continuous and since $\varphi$ is dense in $X, p^{\prime}(x) \geqq p(x)$ for $x \in X$. Thus $X$ is the closed linear span of $\left\{e_{i}\right\}$ in the space $\hat{X}$ with the topology determined by the seminorms $\left\{p^{\prime}: p \in P\right\}$.

For $t \in\left(X X^{r}\right)^{r}$ define

$$
p_{t}(x)=\sup _{n} p\left(\sum_{i=1}^{n} t^{(i)} x^{(i)} e_{i}\right)
$$

Since $t \in\left(X X^{r}\right)^{\gamma},\left\{\sum_{i=1}^{n} t^{(i)} x^{(i)} e_{i}: n=1,2, \cdots\right\}$ is a weakly bounded, thus a strongly bounded subset of $X$ so that

$$
P_{t}(x)<\infty \quad x \in X,
$$

and $p_{t}$ is a continuous seminorm on $X$. If $x \in \hat{X}, t x \in \hat{X}$ and

$$
p^{\prime}(t x)=p_{t}^{\prime}(x)
$$

so that $t \in M_{c}(\hat{X})$. By 3.4, $\left(X X^{r}\right)^{r} \cong M_{c}(X)$. Thus

$$
M_{c}(X) \subseteq M(X) \subseteq\left(X X^{\gamma}\right)^{\beta} \sqsubseteq\left(X X^{\gamma}\right)^{r} \sqsubseteq M_{c}(X)
$$

which establishes the result.

Corollary 5.5. If $\left\{e_{i}, E_{i}\right\}$ is an unconditional basis of a complete barreled space $X$ then

$$
M(X)=\left(X X^{\alpha}\right)^{\alpha}
$$

Proof. Since $\left\{e_{i}, E_{i}\right\}$ is an unconditional basis $X^{\alpha}=X^{\delta}$. If $t \in\left(X X^{r}\right)^{\gamma}$ 
and $u \in X X^{r}$ let $u=x y$ with $x \in X, y \in X^{r}=X^{\delta}=X^{\alpha}$. Let $v^{(i)}=$ sgn $t^{(i)} u^{(i)}$ then $v y \in X^{\alpha}$ so that $v u \in X X^{\gamma}$. Hence, $v u t \in b s$ so that $u t \in l^{1}$. Therefore, $\left(X X^{\gamma}\right)^{\gamma} \subseteq\left(X X^{\alpha}\right)^{\alpha}$ from which the conclusion follows.

THEOREM 5.6. Let $\left\{e_{i}, E_{i}\right\}$ be an absolute basis of a sequentially complete locally convex space $X$. If $P$ is any family of continuous seminorms which determines the topology of $X$ then

$$
M(X)=\left(A A^{\alpha}\right)^{\alpha}
$$

where

$$
A=\left\{\left(p\left(e_{i}\right)\right): p \in P\right\} .
$$

Proof. The hypotheses of this theorem imply that $X=A^{\alpha}$. Now $t \in M(X)$ if and only if $t x \in X$ whenever $x \in X$ which happens if and only if $t x y \in l^{1}$ whenever $x \in A^{\alpha}=X$ and $y \in A$. This will hold if and only if $t \in\left(A A^{\alpha}\right)^{\alpha}$.

EXAMPLES. $\quad M(\omega)=M(\varphi)=\omega ; M(c s)=M\left(b v_{0}\right)=b v ; M\left(c_{0}\right)=M\left(l^{1}\right)=$ $m$.

Let $X$ be the space of all real sequences $x$ for which

$$
p_{k}(x)=\sum_{n}\left|x_{n}\right| k^{n}<\infty \quad k=1,2, \cdots .
$$

Then $X$ with the seminorms $p_{1}, p_{2}, \cdots$ is a nuclear $F$-space which is equivalent to the space of all infinitely differentiable real functions of period $2 \pi$. See $\S 5$ of [6]. If $\left.A=\left(k^{n}\right): k=1,2, \cdots\right\}$ it is clear that $X=A^{\alpha}$. If $x \in A^{\alpha}, x=x e \in A A^{\alpha}$ and if $x \in A A^{\alpha}, x=y\left(k^{n}\right)$ for some $k$ and $y \in A^{\alpha}$. But

$$
\sum_{n=1}^{\infty}\left|t_{n}\right| k^{n} h^{n}=\sum_{n=1}^{\infty}\left|t_{n}\right|(k h)^{n}
$$

so $x \in A^{\alpha}$. Thus $M(X)=A^{\alpha \alpha}=X^{\delta}$.

The following theorem is a version of Theorem 3 and Corollary 2 of [4]. For the definition of $B_{r}$-complete see p. 162 of [9].

Theorem 5.7. A complete biorthogonal system $\left\{e_{i}, E_{i}\right\}$ in a space $X$ which is barreled and $B_{r}$ complete is a basis for $X$ if and only if $M(X) \supseteqq b v$. It is an unconditional basis for $X$ if and only if $M(X) \supseteqq m$.

6. Gamma-perfect BK-algebras. A proper sequential norm $P$ which satisfies the inequality

$$
P(x y) \leqq P(x) P(y)
$$


will be called an algebraic p.s.n. (a.p.s.n.).

THEOREM 6.1. The following statements are equivalent for a sequence space $M$ :

(a) $M$ is a multiplier algebra for a basis in a Banach space;

(b) $M$ is a r-perfect $B K$-algebra containing $e$;

(c) $M=S_{P}$ for $P$ an a.p.s.n. with $P(e)<\infty$.

Proof. (a) $\Rightarrow$ (b). If $M$ is a multiplier algebra for a basis in a Banach space $X$ it is a $B K$-algebra containing $e$ by 3.3 and $\gamma$-perfect since it is the $\gamma$-dual of $X X^{r}$ by 5.4 .

(b) $\Rightarrow(c)$. Suppose $M$ is a $\gamma$-perfect $B K$-algebra containing $e$. By 2.2 (a) there is a sequential norm $Q$ such that $M=S_{Q}$. It is routine to verify that $P$ given by

$$
P(x)=\sup \{Q(x y): Q(y) \leqq 1\}
$$

is a s.n. equivalent to $Q$ (i.e., $S_{P}=S_{Q}$ ) which satisfies (6-1). It remains to show

$$
0<\inf _{n} P\left(e_{n}\right) \leqq \sup _{n} P\left(e_{n}\right)<\infty .
$$

Since $P\left(e_{n}\right)=P\left(e_{n} e_{n}\right) \leqq P\left(e_{n}\right)^{2}$, the left inequality of (6-2) is valid. Since $e \in S_{P}, b s \supseteqq S_{P}^{\gamma}$ so that $b v \subseteq S_{P}$. That $S_{P}$ is $\gamma$-perfect follows form 2.2 (a). The identity map from $b v$ into $S_{P}$ is continuous and $\left\{e_{n}: n=1,2, \cdots\right\}$ is bounded in $S_{P}$. Hence the right inequality in (6-2) is true. Therefore, $P$ is an a.p.s.n.

(c) $\Rightarrow$ (a). If $P$ is an a.p.s.n. with $P(e)<\infty, S_{P}$ is a $B K$-algebra with identity so $M\left(S_{P}\right)=S_{P}$. But since $S_{P}=\left(S_{P}^{0}\right)^{j}$ and $S_{P^{\prime}}=\left(S_{P}^{0}\right)^{\tilde{o}}$, $M\left(S_{P}^{0}\right)=M$ and $\left\{e_{i}, E_{i}\right\}$ is a basis for $S_{P}^{0}(2.2)$.

The following theorem gives a means of constructing $\gamma$-perfect $B K$-algebras with identity which are distinct from $b v$ and $m$. Let $N$ denote the sequence of positive integers and $N_{k}$ a subsequence of the form

$$
N_{k}=\{k(1)<k(2)<\cdots\} .
$$

Theorem 6.2. (a) Let $N_{1}, N_{2}, \cdots, N_{r}$ be a partition of $N$ with each $N_{k}$ given by (6-3). For each $k$ let $P_{k}$ be an a.p.s.n. for which $P_{k}(e)<\infty$. Define $P$ by

$$
P(x)=\max \left\{P_{k}\left(x^{k(1)}, x^{k(2)}, \cdots\right): k=1,2, \cdots r\right\} .
$$

Then $P$ is an a.p.s.n. and $S_{P}$ is a $\gamma$-perfect BK-algebra containing $e$. (b) Let $N_{1}, N_{2}, \cdots$ be an infinite partition of $N$ with each $N_{k}$ given by (6-3). For each $k$ let $P_{k}$ be an a.p.s.n. for which 
Define $P$ by

$$
P(x)=\sup _{k}\left\{P_{k}\left(x^{k(1)}, x^{k(2)}, \cdots\right)\right\} .
$$

Then $P$ is an a.p.s.n. and $S_{P}$ is a $\gamma$-perfect BK-algebra containing $e$.

Proof. The proof of (a) is omitted since it is similar to but less difficult than that of (b).

(b) It is straightforward to verify that $P$ is a norm. That $P$ is a.s.n. follows from the equalities:

$$
\begin{array}{rl}
\sup _{n} & P\left(\sum_{i=1}^{n} x^{(i)} e_{i}\right) \\
& =\sup _{n} \sup _{k}\left\{P_{k}\left(\sum_{k(i) \leqq n} x^{k(i)} e_{i}\right)\right\} \\
& =\sup _{k}\left\{\sup _{n} P_{k}\left(\sum_{k(i) \leqq n} x^{k(i)} e_{i}\right)\right\} \\
& =\sup _{k}\left\{P_{k}\left(x^{k(1)}, x^{k(2)}, \cdots\right)\right\}
\end{array}
$$

since each $P_{k}$ is an s.n. That $S_{P}$ is an algebra follows since

$$
\begin{aligned}
P(x y) & =\sup _{k}\left\{P_{k}\left(x^{k(i)} y^{k(i)}\right)\right\} \\
& \leqq \sup _{k}\left\{P_{k}\left(x^{k(i)}\right) P_{k}\left(y^{k(i)}\right)\right\} \\
& \leqq P(x) P(y) .
\end{aligned}
$$

Therefore, $S_{P}$ is a $\gamma$-perfect $B K$-algebra; it contains $e$ because of (6-4).

EXAmple. Let $M$ be the set of all sequences $x$ such that

$$
P(x)=\sup _{k}\left\{\sum_{i=1}^{\infty}\left|x^{k(i)}-x^{k(i+1)}\right|+\lim _{k(i)}\left|x^{k(i)}\right|\right\}<\infty
$$

for $N_{1}, N_{2}, \cdots$ a partition of the integers with each $N_{k}$ given by (6-3). Then $M$ is a $\gamma$-perfect $B K$-algebra containing $e$ but is neither $m$ nor $b v$. The sequence $y$ with

$$
y^{(i)}=\left\{\begin{array}{l}
1 \text { for } i=k(1) \text { for each } k \\
0 \text { otherwise }
\end{array}\right.
$$

is in $M$ but not in $b v$. The sequence $z$ with

$$
z^{(i)}=\left\{\begin{array}{l}
1 \text { for } i=k(2 j-1) \text { for each } j<k \text { and all } k \\
0 \text { otherwise }
\end{array}\right.
$$

is in $m$ but not $M$.

Question. Are there $\gamma$-perfect $B K$-algebras other than those in 
the smallest class of $B K$-algebras which contain $b v$., and $m$ and are closed under the operations described in Theorem 6.2.?

\section{REFERENCES}

1. J. Diendonné, On biorthogonal systems, Michigan Math. J. 2 (1954), 7-20.

2. N. Dunford and J. T. Schwartz, Linear operators, Part I: General theory, New York, 1958.

3. - Linear operators, Part II Spectral theory, New York, 1963.

4. D. J. H. Garling, Topological sequence spaces (to appear.)

5. G. Köthe and O. Toeplitz, Lineare Räume mit uneudlich vielen Koordinaten und Ringe unendlicher Matrizen, J. für Math. 171 (1934), 193-226.

6. B. S. Mityagin, Approximate dimension and bases in nuclear spaces, Usp. Matem. Nauk. (4) 16 (1961), 63-132.

7. W. Ruckle, On the construction of sequence spaces that have Schauder bases, Canad. J. Math. 18 (1966), 1281-1293.

8. - On the characterization of sequence spaces associated with Schauder bases, Studia Math. 28 (1967), 279-288.

9. H. H. Schaefer, Topological vector spaces, New York, 1966.

10. A. Wilansky, Functional analysis, New York, 1964.

11. S. Yamazaki, Normed rings and bases in Banach spaces, Scientific Papers of the College of General Education, University of Tokyo 15 (1965).

12. - Normed rings and unconditional bases in Banach space, Scientific Papers of the College of General Education 14 (1964).

Received August 5, 1968.

\section{LAFAyette COLlege}

LEHIGH UNIVERSITY 



\section{PACIFIC JOURNAL OF MATHEMATICS}

\section{EDITORS}

H. ROYDEN
Stanford University
Stanford, California

\section{R. R. PHELPS}

University of Washington

Seattle, Washington 98105
J. DUGUNDJI

Department of Mathematics

University of Southern California

Los Angeles, California 90007

\section{RICHARD ARENS}

University of California

Los Angeles, California 90024

\section{ASSOCIATE EDITORS}

E. F. BeCKenbaCh
B. H. NEUMANN

F. WOLF
K. YoshidA

\section{SUPPORTING INSTITUTIONS}

\author{
UNIVERSITY OF BRITISH COLUMBIA \\ CALIFORNIA INSTITUTE OF TECHNOLOGY \\ UNIVERSITY OF CALIFORNIA \\ MONTANA STATE UNIVERSITY \\ UNIVERSITY OF NEVADA \\ NEW MEXICO STATE UNIVERSITY \\ OREGON STATE UNIVERSITY \\ UNIVERSITY OF OREGON \\ OSAKA UNIVERSITY \\ UNIVERSITY OF SOUTHERN CALIFORNIA
}

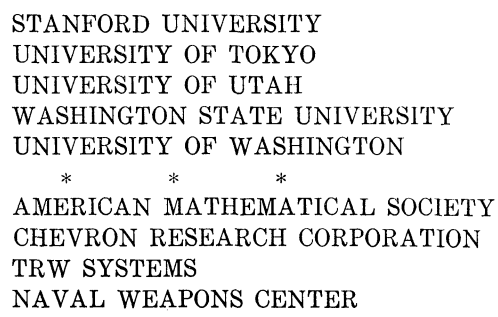

The Supporting Institutions listed above contribute to the cost of publication of this Journal, but they are not owners or publishers and have no responsibility for its content or policies.

Mathematical papers intended for publication in the Pacific Journal of Mathematics should be in typed form or offset-reproduced, double spaced with large margins. Underline Greek letters in red, German in green, and script in blue. The first paragraph or two must be capable of being used separately as a synopsis of the entire paper. It should not contain references to the bibliography. Manuscripts, in duplicate if possible, may be sent to any one of the four editors. Please classify according to the scheme of Math. Rev. 36, 1539-1546. All other communications to the editors should be addressed to the managing editor, Richard Arens, University of California, Los Angeles, California, 90024.

50 reprints are provided free for each article; additional copies may be obtained at cost in multiples of 50 .

The Pacific Journal of Mathematics is published monthly. Effective with Volume 16 the price per volume (3 numbers) is $\$ 8.00$; single issues, $\$ 3.00$. Special price for current issues to individual faculty members of supporting institutions and to individual members of the American Mathematical Society: $\$ 4.00$ per volume; single issues $\$ 1.50$. Back numbers are available.

Subscriptions, orders for back numbers, and changes of address should be sent to Pacific Journal of Mathematics, 103 Highland Boulevard, Berkeley, California, 94708.

PUBLISHED BY PACIFIC JOURNAL OF MATHEMATICS, A NON-PROFIT CORPORATION

Printed at Kokusai Bunken Insatsusha (International Academic Printing Co., Ltd.), 7-17, Fujimi 2-chome, Chiyoda-ku, Tokyo, Japan. 


\section{Pacific Journal of Mathematics \\ Vol. 29, No. $2 \quad$ June, 1969}

Bruce Langworthy Chalmers, On boundary behavior of the Bergman kernel function and related domain functionals ................... 243

William Eugene Coppage, Peirce decomposition in simple Lie-admissible power-associative rings .............................. 251

Edwin Duda, Compactness of mappings...................... 259

Earl F. Ecklund Jr., On prime divisors of the binomial coefficient......... 267

Don E. Edmondson, A modular topological lattice ............... 271

Phillip Alan Griffith, A note on a theorem of Hill ................... 279

Marcel Herzog, On finite groups with independent cyclic Sylow

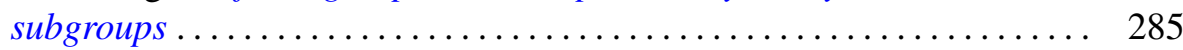

James A. Huckaba, Extensions of pseudo-valuations................. 295

S. A. Huq, Semivarieties and subfunctors of the identity functor ........ 303

I. Martin (Irving) Isaacs and Donald Steven Passman, Finite groups with small character degrees and large prime divisors. II ............ 311

Carl Kallina, A Green's function approach to perturbations of periodic

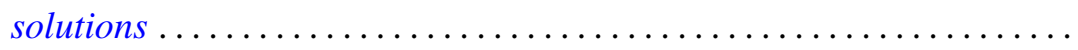

$\mathrm{Al}$ (Allen Frederick) Kelley, Jr., Analytic two-dimensional subcenter manifolds for systems with an integral ....................

Alistair H. Lachlan, Initial segments of one-one degrees ............ 351

Marion-Josephine Lim, Rank k Grassmann products ............. 367

Raymond J. McGivney and William Henry Ruckle, Multiplier algebras of

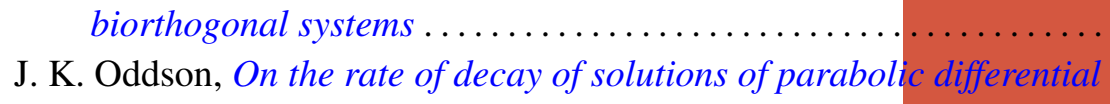

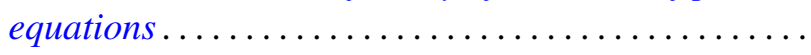

Helmut R. Salzmann, Geometries on surfaces ........... .

Annemarie Schlette, Artinian, almost abelian groups and their groups of automorphisms ............................

Edgar Lee Stout, Additional results on modules over polydisc algebras ...

Lajos Tamássy, A characteristic property of the sphere . .

Mark Lawrence Teply, Some aspects of Goldie's torsion theory. ...

Freddie Eugene Tidmore, Extremal structure of star-shaped sets ...

461

Leon Jarome Weill, Unconditional and shrinking bases in locally convex spaces... 